\title{
Physikalische Therapie: Asthma bronchiale
}

\author{
Jean-Michel Jeannin
}

Basel, Schweiz

\section{Einleitung}

Asthma bronchiale ist eine häufige chronische Krankheit der Atemwege, namentlich der Bronchien. In der Schweiz z.B. sind $8 \%$ der Bevölkerung betroffen [1]. Es handelt sich um eine ausgesprochen individuell geprägte Krankheit mit einer vielfältigen Ausformung. Entsprechend sind auch individuelle Behandlungsmethoden gefragt. Die Behandlung des Asthma bronchiale befindet sich in einem Spannungsfeld zwischen der etablierten medikamentösen Therapie mit Bronchodilatatoren und Kortikosteroiden und einer Vielfalt von Behandlungsmodalitäten der physikalischen Therapie sowie der Komplementärmedizin. Die Wirksamkeit von Arzneimitteln lässt sich nach den strengsten Bedingungen nachweisen, ist aber nur auf wenige Zielparameter beschränkt. Die physikalische Therapie setzt auf eine grössere Breite, ist eher ganzheitlich und pleiotrop, jedoch lässt sich ihre Wirksamkeit in placebokontrollierten Studien nicht immer gleich gut belegen. Diesem Spannungsfeld wird nachfolgend nachgegangen.

\section{Asthma bronchiale}

Asthma bronchiale ist trotz seines häufigen Vorkommens eine nur ungenau definierte Krankheit. Zu dieser Schwierigkeit mag auch die Tatsache beitragen, dass Asthma bronchiale auch Symptom einer Grundkrankheit sein kann. Dazu zählen die Atopie (allergisches Asthma), Bronchiektasien und chronisch obstruktive Lungenkrankheiten [2]. Die Weltgesundheitsorganisation definiert Asthma bronchiale als eine chronische, durch rekurrierende Anfälle von Atemnot und pfeifendem Atem charakterisierte Krankheit. Die Symptome können mehrmals am Tag bzw. in der Woche auftreten und können je nach Individuum bei körperlicher Tätigkeit oder in der Nacht verstärkt auftreten. Ein Asthma-Anfall wird durch das
Anschwellen der Bronchialschleimhaut und die dadurch verursachte Verengung der Atemwege ausgelöst. Häufige Asthma-Anfälle sind für Schlaflosigkeit, Müdigkeit, Antriebsverlust und Fehlen in der Schule oder am Arbeitsplatz verantwortlich [3]. Asthma galt längere Zeit als klassische psychosomatische Krankheit. Betroffene leiden überdurchschnittlich häufig an einer Depression oder Angststörung. Aktuell werden eine Störung der Stressachse, des Immunsystems und des autonomen Nervensystems als gemeinsame Grundlage betrachtet [4]. Bei dieser Betrachtungsweise ist die gegenseitige Beeinflussung von psychischen und somatischen Faktoren besser berücksichtigt. Während psychischer Stress den Atemwegswiderstand von nicht von Asthma betroffenen Patienten senkt, vergrössert er ihn bei Asthma-Patienten [5]. Wie viele chronische Krankheiten beeinträchtigt auch Asthma bronchiale die Lebensqualität der Betroffenen in einem bisweilen erheblichen Mass. Die multifaktorielle Ätiologie umfasst genetische Faktoren, Umweltfaktoren und entzündliche Komponenten [6]. In der Regel führt körperliche Anstrengung zu einer Bronchodilatation, bei einigen Individuen dagegen zu einer Bronchokonstriktion. An einer belastungsindizierten Bronchokonstriktion können sowohl von Asthma Betroffene als auch Nichtbetroffene leiden [7]. Spitzensportler sind überdurchschnittlich häufig betroffen, unabhängig davon, ob sie gleichzeitig auch an Asthma bronchiale leiden [8]. Im Grossen und Ganzen ist die Behandlung mit derjenigen des Asthma bronchiale identisch [7].

\section{Notwendigkeit von nichtmedikamentösen Therapien}

Die medikamentöse Therapie besteht aus der Verabreichung von Kortikosteroiden, Leukotrienrezeptor-Antagonisten und Bronchodilatatoren [6]. Sie ist sehr wirksam, betrifft aber nur wenige Symptome, ist nicht nach-

\section{KARGER}

Fax +497614520714 Information@Karger.com www.karger.com

\section{(c) 2017 S. Karger GmbH, Freiburg}

Accessible online at:

www.karger.com/szg 


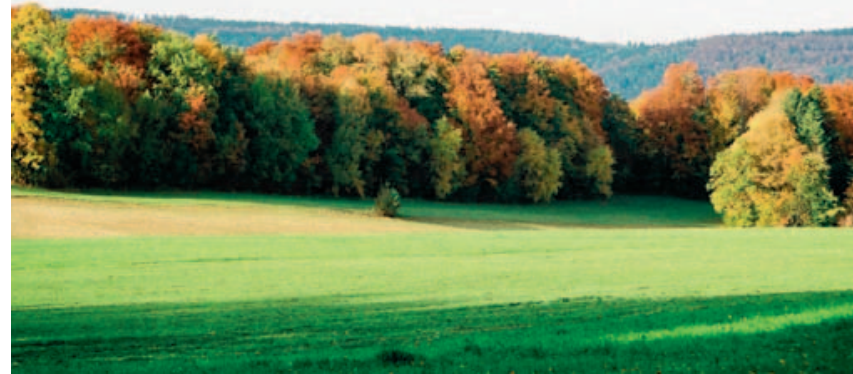

Abb. 1. Wald bei Pleigne JU: Saubere Luft (Expositionsprophylaxe) sowie Licht und Farbe (antidepressive Wirkung).

haltig und ist insgesamt eingeschränkt. Die verschiedenen Therapiemittel der physikalischen Therapie wirken hingegen umfassend, schliessen sie doch auch atemmechanische, psychische und soziale Aspekte ein, und zudem präventiv. Nichtmedikamentöse Therapien sind auch aus sozioökonomischen Gründen notwendig, da grosse Teile der betroffenen Weltbevölkerung die Medikamente nicht zu bezahlen vermag [6]. Die wichtigsten Methoden der physikalischen Therapie für die Behandlung des Asthma bronchiale sind Ausdauerleistungstraining [9], Atmungstherapie, Atemgymnastik und Inhalationstherapie, ferner auch Klimatherapie [10].

\section{Klinische Erfahrung}

Huntley et al. [5] werteten 15 Publikationen von klinischen Studien zu Entspannungstherapien bei Asthma bronchiale aus. In 9 davon wurde die Intervention gegenüber einer adäquaten Vergleichsgruppe bewertet. Muskelentspannungstherapien vermochten die Lungenfunktion von Asthma-Patienten verbessern, nicht aber Transzendentale Meditation, Autogenes Training, Hypnotherapie und Biofeedback-Übungen.

Carson et al. [10] selektierten aus 1939 Studien zur Wirksamkeit von Trainingsprogrammen bei Asthma bronchiale 21 Studien mit insgesamt 722 Teilnehmern. Das Training wurde als aerobe, den ganzen Körper umfassende Übungen definiert, die zweimal wöchentlich während mindestens 20 min über mindestens 4 Wochen durchzuführen waren. In keiner der Studien wurde eine Verschlechterung des Asthmas nach den Trainingsübungen beobachtet. Anhand der maximalen Sauerstoffaufnahme wurde eine deutliche Verbesserung des kardiopulmonalen Leistungsvermögens («cardiopulmonal fitness») festgestellt. Die Lungenfunktionsparameter Einsekun-

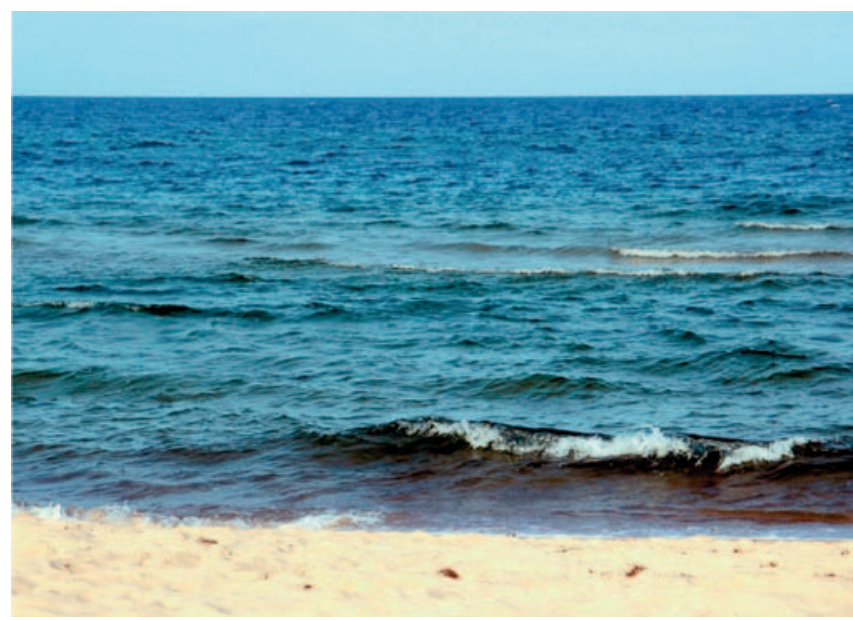

Abb. 2. Ostseestrand (Rügen): Klimatherapie und Expositionsprophylaxe.

denvolumen, forcierte Vitalkapazität und Ausatmungsspitzenfluss (peak expiratory flow rate) wurden dagegen nicht verändert. Auch wenn die Daten aus den einzelnen Studien nicht direkt miteinander verglichen werden konnten, sehen die Autoren eine gewisse Evidenz dafür, dass Trainingsübungen bei Asthma-Patienten einen positiven Effekt auf die Lebensqualität ausüben.

Pakhale et al. [6] untersuchten in einer Übersichtsarbeit 23 Studien, die sie aus einer Gesamtheit von 2635 Studien selektierten und die zum Teil mit denjenigen identisch waren, die Carson et al. [10] bewertet hatten. Die Autoren verglichen hierbei die Effekte von Trainingsübungen auf das C-reaktive Protein (CRP), auf Immunglobulin E (IgE), auf Stickstoffmonoxid, auf die Anzahl der Leukozyten im Sputum sowie auf die Reagibilität der Bronchien. Es wurde eine Verminderung des CRP, des Stickstoffmonoxids, der Anzahl der Zellen im Sputum und des IgE festgestellt. Der Einfluss der Behandlung auf die Reagibilität war in den verschiedenen Studien unterschiedlich dargestellt. Die Art, Intensität, Frequenz und Dauer des Trainings waren uneinheitlich definiert, ebenso das Studiendesign. Die Autoren fanden Anhaltspunkte dafür, dass körperliche Tätigkeit die Entzündung der Atemwege bei Asthma-Patienten lindert.

Bei der Verordnung einer Klimatherapie muss die kardiopulmonale Leistungsfähigkeit des Patienten beachtet werden. Für Patienten, die schon im Flachland (Abb. 1) eine Verminderung der Sauerstoffsättigung des Blutes aufweisen, ist eine Hochgebirgsklimatherapie kontraindiziert. Eine Kur an der Nordsee kann hingegen sinnvoll und effektiv sein: Ein 10-jähriger übergewichtiger Junge muss zu Beginn der Therapie bis zu fünfmal am Tag Bronchospasmolytika inhalieren. Zum Programm gehören Klimaexpositionen am Meer, deren Dauer zunimmt (Abb. 2). Es folgen Bäder im Meer bis zu den Knien und schliesslich Ganzkörperbäder. Spiel und Wanderungen 
am Strand steigern die Klimaexposition ein weiteres Mal. Nach 4 Wochen Therapie benötigt der Patient Bronchospasmolytika nur noch zweimal am Tag; seine Bewegungsaktivität und die kardiopulmonale Leistungsfähigkeit sind stark gesteigert [11].

\section{Reflexionen}

Trotz allen Fortschritten bezüglich seiner Erforschung bleibt das Asthma bronchiale eine geheimnisvolle Krankheit mit einer ausgesprochen individuellen Ausprägung. Die Mittel der physikalischen Therapie eignen sich sehr gut für sehr fein auf das Individuum abgestimmte Behandlungen. Die physikalische Therapie ist demnach zur Behandlung des Asthma bronchiale sehr gut geeignet. Die medikamentöse Therapie zielt auf nur wenige Parameter ab. Die Wirksamkeit eines Medikaments lässt sich mittels randomisierten, placebogesteuerten klinischen Studien statistisch gesichert nachweisen. Die Wirksamkeitsnachweise für Behandlungsmethoden der physikalischen Therapie sind komplexer, weil die Interventionen komplexer als die Verabreichung eines Medikaments sind und sie eine Vielzahl von Zielparametern angreifen. Wie die angeführten Beispiele zeigen, ist es dennoch möglich, die Wirksamkeit von ausgewählten Interventionen auf ausgewählte Zielparameter statistisch gesichert nachzuweisen.

In placebokontrollierten klinischen Studien wird der Nettobeitrag eines Medikaments oder die Wirkung einer physikalischen Therapie auf einen definierten Zielparameter an einer Behandlung gemessen. Hierbei wird eine Reihe von Behandlungsmomenten ausgeblendet, die nicht einfach gemessen werden können: die Zuwendung der therapeutisch agierenden Person, die Verbesserung des Selbstbewusstseins des Patienten, eine Verbesserung der Stimmung, soziale Kontakte, die Verbesserung der Selbststeuerung, die Anregung der Selbstheilungskräfte. Solche Wirkungen lassen sich mit einer umfassenden, ganzheitlichen Therapie besser erzielen als allein mit Medikamenten. Sie werden noch viel zu oft als «Placeboeffekte» bezeichnet und abgewertet. Statt einer Abwertung wäre es vernünftiger, neue Techniken zur Bewertung von Behandlungen und Behandlungsmethoden zu entwickeln, wie es schon hin und wieder der Fall ist.

\section{Literatur}

1 Bronchial asthma; in WHO Fact Sheet $\mathrm{N}^{\circ}$ 206. http://who.int/mediacentre/factsheets/ fs206/en.

2 Hargreave FE, Nair P: The definition and diagnosis of asthma. Clin Exp Allergy 2009;39: 1652-1658.

3 Asthma; in WHO Fact Sheet $\mathrm{N}^{\circ} 307$. http:// who.int/mediacentre/factsheets/fs307/en.

4 Lieshout RJV, MacQueen GM: Relations between asthma and psychological distress: an old idea revisited. Chem Immunol Allergy 2012;98:1-13.
5 Huntley A, White AR, Ernst E: Relaxation therapies for asthma: a systematic review. Thorax 2002;57:127-131.

6 Pakhale S, Luks V, Burkett A, Turner L: Effect of physical training on airway inflammation in bronchial asthma. BMC Pulm Med 2013, 13:38.

7 Jayasinghe H, Kopsaftis Z, Carson K: Asthma bronchiale and exercise-induced bronchoconstriction. Respiration 2015;89:505-512.

8 Boulet LP, O'Byrne PM: Asthma and exercise-induced bronchoconstriction in athletes. N Engl J Med 2015;372:641-648.
9 Gutenbrunner C, Glaesener JJ: Krankengymnastische Techniken; in Gutenbrunner C, Glaesener JJ (Hrsg): Rehabilitation, Physikalische Medizin und Heilverfahren. Heidelberg, Springer, 2007, p 34.

10 Carson KV, Chandratilleke MG, Picot J, et al: Physical training for asthma (review). Cochrane Database Syst Rev 2013;(9): CD001116.

11 Schuh A: Klimatherapie; in: Gutenbrunner C, Glaesener JJ (Hrsg): Rehabilitation, Physikalische Medizin und Heilverfahren. Heidelberg, Springer, 2007, p 90. 